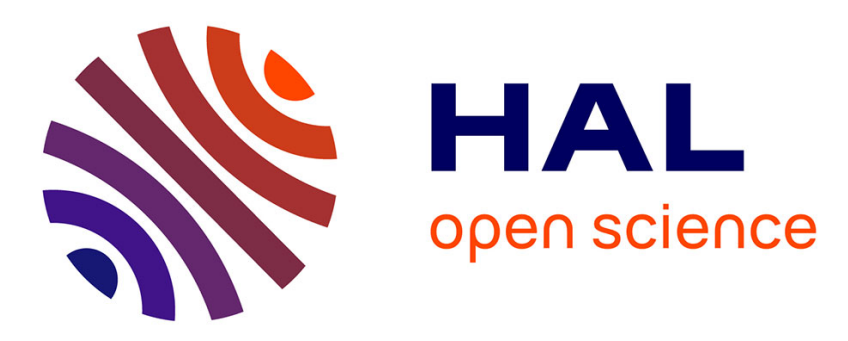

\title{
Oscillatory-nonoscillatory transitions for inclined cellular patterns in three-dimensional directional solidification
}

\author{
Fatima L Mota, Nathalie Bergeon, Alain Karma, Rohit Trivedi, Jean-Marc
}

Debierre

\section{- To cite this version:}

Fatima L Mota, Nathalie Bergeon, Alain Karma, Rohit Trivedi, Jean-Marc Debierre. Oscillatorynonoscillatory transitions for inclined cellular patterns in three-dimensional directional solidification. Physical Review E , 2020, 102 (3), pp.032803. 10.1103/PhysRevE.102.032803 . hal-02948833

\section{HAL Id: hal-02948833 \\ https://hal.science/hal-02948833}

Submitted on 25 Sep 2020

HAL is a multi-disciplinary open access archive for the deposit and dissemination of scientific research documents, whether they are published or not. The documents may come from teaching and research institutions in France or abroad, or from public or private research centers.
L'archive ouverte pluridisciplinaire HAL, est destinée au dépôt et à la diffusion de documents scientifiques de niveau recherche, publiés ou non, émanant des établissements d'enseignement et de recherche français ou étrangers, des laboratoires publics ou privés. 


\title{
Oscillatory-nonoscillatory transitions for inclined cellular patterns in three-dimensional directional solidification
}

\author{
Fatima L. Mota $\odot,{ }^{1, *}$ Nathalie Bergeon, ${ }^{1}$ Alain Karma, ${ }^{2}$ Rohit Trivedi, ${ }^{3}$ and Jean-Marc Debierre ${ }^{1}$ \\ ${ }^{1}$ Aix-Marseille Université, CNRS, Université de Toulon, IM2NP UMR 7334, 13397 Marseille, France \\ ${ }^{2}$ Department of Physics and Center for Interdisciplinary Research on Complex Systems, Northeastern University, \\ Boston, Massachusetts 02115 USA \\ ${ }^{3}$ Department of Materials Science and Engineering, Iowa State University, Ames, Iowa 50010, USA
}

(Received 16 July 2020; accepted 8 September 2020; published xxxxxxxxxx)

\begin{abstract}
The oscillatory behavior of cellular patterns produced by directional solidification of a transparent alloy under microgravity conditions was recently observed to depend on the misorientation of the main crystal axis with respect to the direction of the imposed thermal gradient [Pereda et al., Phys. Rev. E 95, 012803 (2017)]. To characterize the oscillatory-nonoscillatory transition resulting from the variations of the crystal misorientation, new experiments performed in DECLIC-DSI onboard the International Space Station and phase-field simulations are analyzed and combined in the present study. Experimental results are extracted from movies showing regions that extend on both sides of a boundary between two grains with respective misorientations of roughly 3 and 7 degrees. A set of tools are developed to analyze the experimental data and the same analysis is reproduced for the numerical data. A number of points are addressed in the simulations, like the effects of the system dimensions. The oscillatory state is found to be favored by the increase of the geometrical degrees of freedom. In bulk samples, a good agreement is found between the experimental and the numerical oscillatory-nonoscillatory threshold given by the ratio of the drift time to the oscillation period at the transition. The existence and the origin of bursts of localized groups of oscillating cells within a globally nonoscillatory pattern are characterized. A qualitative description of the physical mechanism that governs the oscillatory-nonoscillatory transition is provided.
\end{abstract}

DOI: 10.1103/PhysRevE.00.002800

\section{INTRODUCTION}

In the recent decades, a number of solidification experiments led to spectacular oscillatory growth modes for both liquid crystals [1] and eutectic alloys [2,3]. Comparable oscillatory patterns were also observed in directional solidification of succinonitrile-based diluted binary alloys [4-8]. In thin samples, the existence of a generic $2 \lambda-O$ mode was reported and characterized by a large set of experimental and numerical data [4,5]. More recently, it was shown that these oscillations result from a coherent interaction between the solute diffusion fields that surrounds two neighboring cell tips (firstneighbor correlations) [5]. In bulk samples, oscillating growth modes are more difficult to observe experimentally and their existence was not mentioned until recent experiments were performed under microgravity conditions in the DECLIC-DSI device. These experiments produced arrays of oscillating cells without global coherence but still displaying locally coherent oscillations with a $\pi$ phase-shift in the case of local square ordering or a $2 \pi / 3$ phase-shift in the case of local hexagonal ordering [6-8]. These coherent spatiotemporal oscillating patterns are known to result from generic 2D bifurcations [9].

Several studies based on numerical simulations of the corresponding oscillation dynamics can be found in the literature. For thin samples, a first study using the one-sided phase field

*Corresponding author: fatima.lisboa-mota@im2np.fr model reported the onset of an oscillatory single structure in an intermediate domain of spacings [10]. Subsequently, a similar model was used in a dedicated study of $2 \lambda-\mathrm{O}$ mode that gave rather quantitative agreement with the oscillations existence domain and proposed a complete description of the physical underlying mechanisms [5]. For bulk samples, numerical studies first relied on amplitude equations [11-13], later on the two-sided version of the phase-field formalism applied to a model system [14], and even more recently on the one-sided phase-field model parametrized with the actual physical parameters of microgravity experiments [6-8]. These simulations also generated $\pi$ and $2 \pi / 3$ oscillatory modes with oscillation periods comparable to the experimental ones [6-8]. In addition, they showed that, for a given alloy, oscillations only exist in a limited domain of spacings, pulling velocities, and temperature gradients. A recent analysis of a series of DECLIC experimental data suggested that cell oscillations also stop when the misorientation of the $\langle 100\rangle$ crystal axis with respect to the thermal gradient (parallel to the pulling direction) becomes too large [8]. A criterion was proposed to evaluate the critical inclination above which oscillations stop and a rough estimate of this threshold was obtained [8]. The main goal of the present paper is to more finely characterize and quantify the threshold for this oscillatory-nonoscillatory transition due to the crystal misorientation. This problematic is attacked by applying a common methodology to analyze new experimental and phase-field simulation data and by comparing the resulting informations. 
The present phase-field approach readily combines two numerical codes used in recent studies that considered crystal misorientation and oscillatory states separately. In both cases, solidification of a succinonitrile-acrylonitrile alloy was simulated in a thin sample and the simulation results were compared with experimental data $[5,15]$. On the one hand, regarding the effects of the crystal misorientation, the numerical results [15] confirmed the orientational response law found experimentally $[16,17]$. Since this study focused on two specific growth shapes, steady cells and seaweeds, no particular attention was paid to the possibility of oscillating structures. On the other hand, the study of a well-oriented $2 \lambda-\mathrm{O}$ oscillatory mode showed that quantitative results can be obtained for its existence domain and oscillation period [5]. This study also demonstrated the benefits that can be drawn from numerical data obtained for the concentration field, to follow the coupled dynamics of first-neighbor cells. Altogether, combining both approaches is thus expected to provide a reliable and powerful tool to explore the oscillatory-nonoscillatory transition of inclined cellular patterns in three-dimensional directional solidification.

We report here on new experimental results and on numerical simulations of the oscillatory-nonoscillatory transition. A number of points are addressed, like a comparison between thin layer and bulk samples. So far, a direct experimental comparison could not be attempted between the two cases because a different solute was used, acrylonitrile in thin samples [4,16] and camphor in the bulk samples [6-8]. It is one nice feature of the present numerical simulations to give results that are obtained in both geometries for the same alloy (succinonitrile-camphor is considered here). This paper is organized as follows. Section II describes the experimental procedure and the main results obtained, while Sec. III gives the basic ingredients of the phase-field code used in the simulations. Section IIIC is devoted to the thin layer samples. The results for the bulk samples are given and analyzed in Sec. III D. Finally, a discussion completed by our main conclusions is provided in Sec. IV.

\section{EXPERIMENTAL}

\section{A. Directional solidification experiments in DECLIC-DSI: Device, method, and parameters}

The experiment described in this article was realized within the directional solidification insert (DSI) of the device for the study of critical liquids and crystallization (DECLIC) developed by the French Space Agency (CNES) and installed onboard the International Space Station (ISS). This device is dedicated to in situ and real-time characterization of the solid-liquid interface during directional solidification of bulk transparent alloys in diffusive transport conditions. Complete descriptions of DECLIC and DSI can be found elsewhere [18-20]. In DECLIC-DSI, a Bridgman furnace imposes a thermal gradient $G$ in the interfacial area of the solidifying alloy contained in a glass crucible. Solidification is induced by pulling the crucible at constant rate $V_{p}$ towards the cold side on the furnace. The interface is observed from the top, i.e., along the direction of solidification, thanks to a flat glass window at the bottom of the crucible and a lens immersed in the liquid at the top. The transparent organic alloy used
TABLE I. Physical parameters of the succinonotrile-camphor alloy of interest and control parameters imposed in the experiments.

\begin{tabular}{lccc}
\hline \hline Physical parameter & Symbol & Magnitude & Unit \\
\hline Liquidus slope & $m$ & -1.365 & $\mathrm{~K} / \mathrm{wt} \%$ \\
Solute (camphor) concentration & $c_{\infty}$ & 0.24 & $\mathrm{wt} \%$ \\
Solute diffusion coefficient (liq.) & $D$ & 270.0 & $\mu \mathrm{m}^{2} / \mathrm{s}$ \\
Gibbs-Thomson coefficient & $\Gamma$ & 0.06478 & $\mathrm{~K} \mu \mathrm{m}$ \\
Partition coefficient & $k$ & 0.07 & - \\
Anisotropy strength & $\epsilon_{4}$ & 0.011 & - \\
\hline Control parameter & Symbol & Magnitude & Unit \\
\hline Thermal gradient & $G$ & 19 & $\mathrm{~K} / \mathrm{cm}$ \\
Pulling velocity & $V_{p}$ & 0.75 or 1.5 & $\mu \mathrm{m} / \mathrm{s}$ \\
\hline \hline
\end{tabular}

is a succinonitrile $(\mathrm{SCN})-0.24 \mathrm{wt} \%$ camphor alloy prepared using SCN purified by NASA by distillation and zone melting (Table I). The cylindrical crucible has an inner diameter of $1 \mathrm{~cm}$ and a length that enables about $10 \mathrm{~cm}$ of solidification, thus allowing the study of the interface pattern dynamics from the onset of morphological instability to the microstructural steady state. A single crystalline solid seed with a direction $\langle 100\rangle$ parallel to the pulling axial direction was prepared on ground and kept during all the experimental campaigns. Further details about the experimental procedure can be found in previous works [18-21].

Top-view images are used to morphologically characterize the patterns developed during microgravity experiments. Methods of analysis $[8,22]$ start from the raw image where a binary mask is created to identify and label each cell and its center. Based on this image, a Voronoi tessellation reliably finds the first neighbors at each instant of the solidification, so that maps of the number of first neighbors can be drawn, to get information on the quantity of topological defects in the pattern. The Voronoi tessellation is used to calculate the primary spacing which corresponds to the average of the center-tocenter distances between a cell and its first-neighbors. Each cell is tracked from one image to the following one so that its motion can be analyzed (trajectory, velocity), and elimination or nucleation of new cells is evidenced.

During spatial experiments, two different values of gradients and a wide range of pulling rate, from 0.1 to $30 \mu \mathrm{m} / \mathrm{s}$, were used. We could therefore study various complex patterns, from planar to cellular and dendritic. However, the occurrence of oscillating cellular patterns is restricted to a very limited range of experimental parameters which are detailed in Ref. [8]. Most of the oscillating patterns are obtained for $G=19 \mathrm{~K} / \mathrm{cm}$, and for $V_{p}$ ranging between 0.5 and $1.5 \mu \mathrm{m} / \mathrm{s}$. In this paper, we will deepen the analysis of two solidification experiments for a temperature gradient $G=19 \mathrm{~K} / \mathrm{cm}$, and pulling velocities $V_{p}=0.75$ and $1.5 \mu \mathrm{m} / \mathrm{s}$, that yield oscillations of a cellular array, but also specifically evidence the influence of misorientation on the oscillations (Table I). As was explained in Ref. [8], for $V_{p}=1.0$ and $0.5 \mu \mathrm{m} / \mathrm{s}$, once oscillations start, they affect the whole interface for the remainder of the experiment. For $V_{p}=0.75$ and $1.5 \mu \mathrm{m} / \mathrm{s}$, oscillations initially affect the whole interface but after the sample is pulled by $L \simeq 30 \mathrm{~mm}$, cells on the left side 

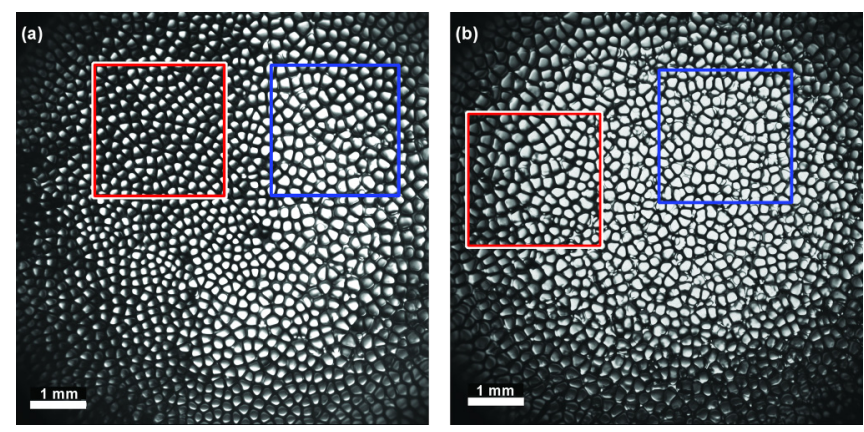

FIG. 1. Global view of the interface: (a) at $t=4.38 \mathrm{~h}(L=$ $24 \mathrm{~mm}$ ) for $V_{p}=1.5 \mu \mathrm{m} / \mathrm{s}$ and (b) at $t=8.12 \mathrm{~h}(L=22 \mathrm{~mm})$ for $V_{p}=0.75 \mu \mathrm{m} / \mathrm{s}$ and $G=19 \mathrm{~K} / \mathrm{cm}$. The red and blue squares (white and black in the grayscale version) correspond to the analysis regions on the left and right side of the interface, respectively.

of the interface stop oscillating, while oscillations continue on the right side [8]. The origin for the premature termination of the left-side oscillation is attributed to the consequencesin terms of pattern drift - of a misorientation of the growth direction in this area, as will be detailed in the next part. For $V_{p}=1.5 \mu \mathrm{m} / \mathrm{s}$, oscillations on the right side also stop shortly after because the pulling velocity that keeps increasing due to transient growth reaches the limit of the existence domain of oscillations [8]: No oscillation appears for velocities higher than $1.5 \mu \mathrm{m} / \mathrm{s}$.

\section{B. Evidence and characterization of growth misorientations}

Figure 1 shows a global view of the solid-liquid interface after about $20 \mathrm{~mm}$ of solidification for both pulling rates. For $V_{p}=1.5 \mu \mathrm{m} / \mathrm{s}$, a clear morphological difference is observed between the left region (red-or white—square) that displays inclined structures and the right region (blue-or blacksquare) that does not. The origin of inclined structures is a misalignment between the preferred growth direction $\langle 100\rangle$ and the pulling (thermal) axis [16,17]: the growth direction of a cell or dendrite rotates from the thermal gradient direction at low velocity, to the closest $\langle 100\rangle$ direction as pulling rate increases. This implies that the growth direction will be misoriented with respect to the pulling (thermal) axis if no $\langle 100\rangle$ direction is perfectly aligned with this axis. If the misalignment between the preferred growth direction $\langle 100\rangle$ and the pulling (thermal) axis is large enough, then structures are tilted with respect to the optical axis, and we can distinguish subgrains by visual inspection of the microstructure. The presence of at least one subboundary in Fig. 1 is clear for $V_{p}=1.5 \mu \mathrm{m} / \mathrm{s}$. To confirm it, it is useful to analyze the cell trajectories: each subgrain is characterized by a collective cell drift of specific direction and amplitude, caused by its particular misalignment with the pulling (thermal) axis. For $V_{p}=1.5 \mu \mathrm{m} / \mathrm{s}$, the cell drift velocities are mapped in Fig. 2, both in direction and module: On the left side of the interface, the drift velocity is almost twice higher than on the right side, confirming the presence of a subboundary. We may note that, in the lower left area, the drift directions are different from the ones in the upper left area, suggesting a second boundary that splits the left part into two subgrains. As we never remelted the initial single crystal of selected orientation
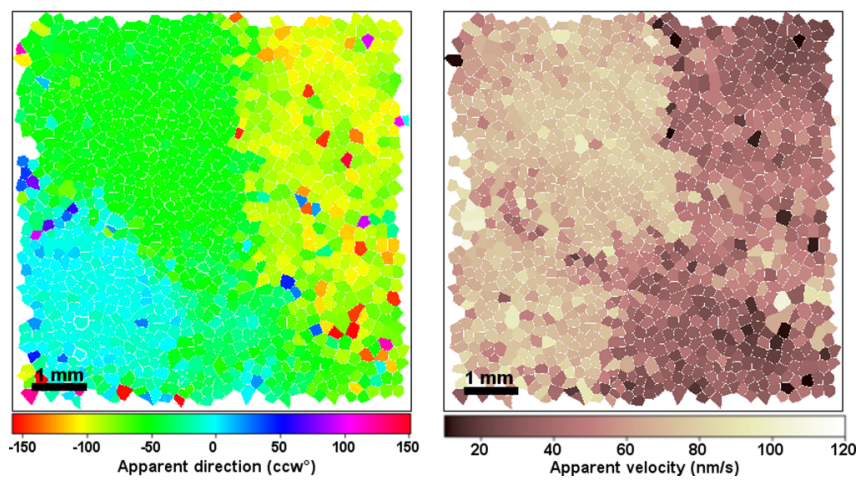

FIG. 2. Maps of the apparent drift (a) direction and (b) velocity of each cell $\left(V_{p}=1.5 \mu \mathrm{m} / \mathrm{s}, G=19 \mathrm{~K} / \mathrm{cm}, t=4.62 \mathrm{~h}, L=25 \mathrm{~mm}\right)$.

during the whole experimental campaign, the formation of subboundaries is attributed to dislocation reorganizations into subboundaries, and thus to a polygonization of the seed crystal, due to successive thermal cycles. For $V_{p}=0.75 \mu \mathrm{m} / \mathrm{s}$, it is difficult to spot any subboundary from Fig. 1. Despite a general heterogeneity on the right side, throughout the analysis of cell trajectories, one clearly distinguish a group of cells moving in the same direction and with the same velocity on the left side of the interface, whereas on the right side the cells are drifting with a velocity more than twice lower, confirming the presence of the subboundary between left and right.

Focusing on the regions identified in Fig. 1, the mean drift velocity evolution is shown in Figs. 3(a) and 3(c). The amplitude of the drift velocity $V_{d}$ can be used to estimate the tilt angle $\theta_{g}$ between the growth velocity and the pulling velocity, considering a macroscopically flat interface:

$$
\tan \theta_{g}=V_{d} / V_{p}
$$
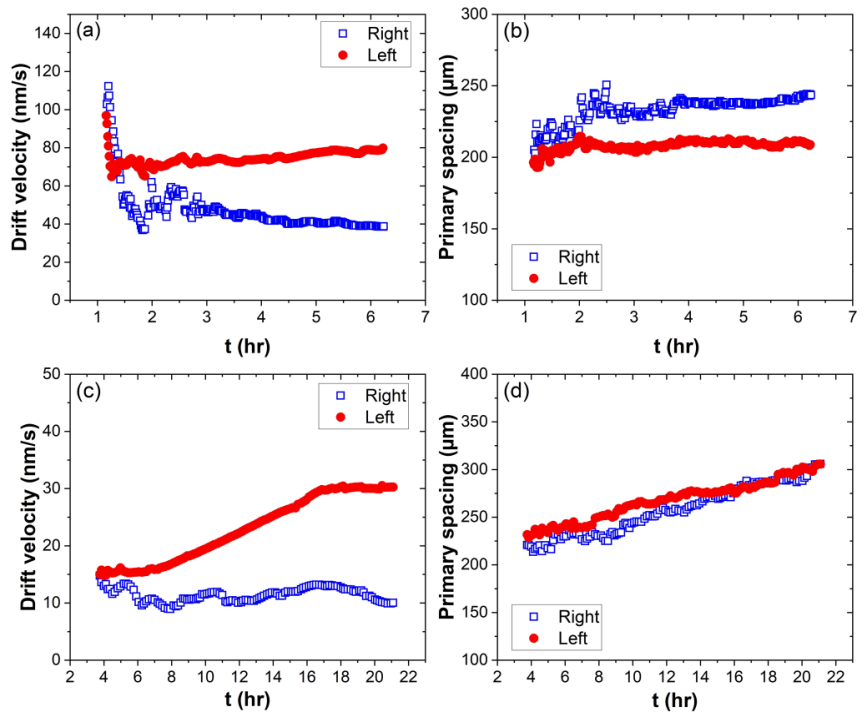

FIG. 3. Evolution of (a), (c) the drift velocity and (b), (d) the primary spacing as functions of the solidification time for the two regions identified in Fig. 1: $G=19 \mathrm{~K} / \mathrm{cm} ; V_{p}=1.5 \mu \mathrm{m} / \mathrm{s}$ (upper panels); and $V_{p}=0.75 \mu \mathrm{m} / \mathrm{s}$ (lower panels). 

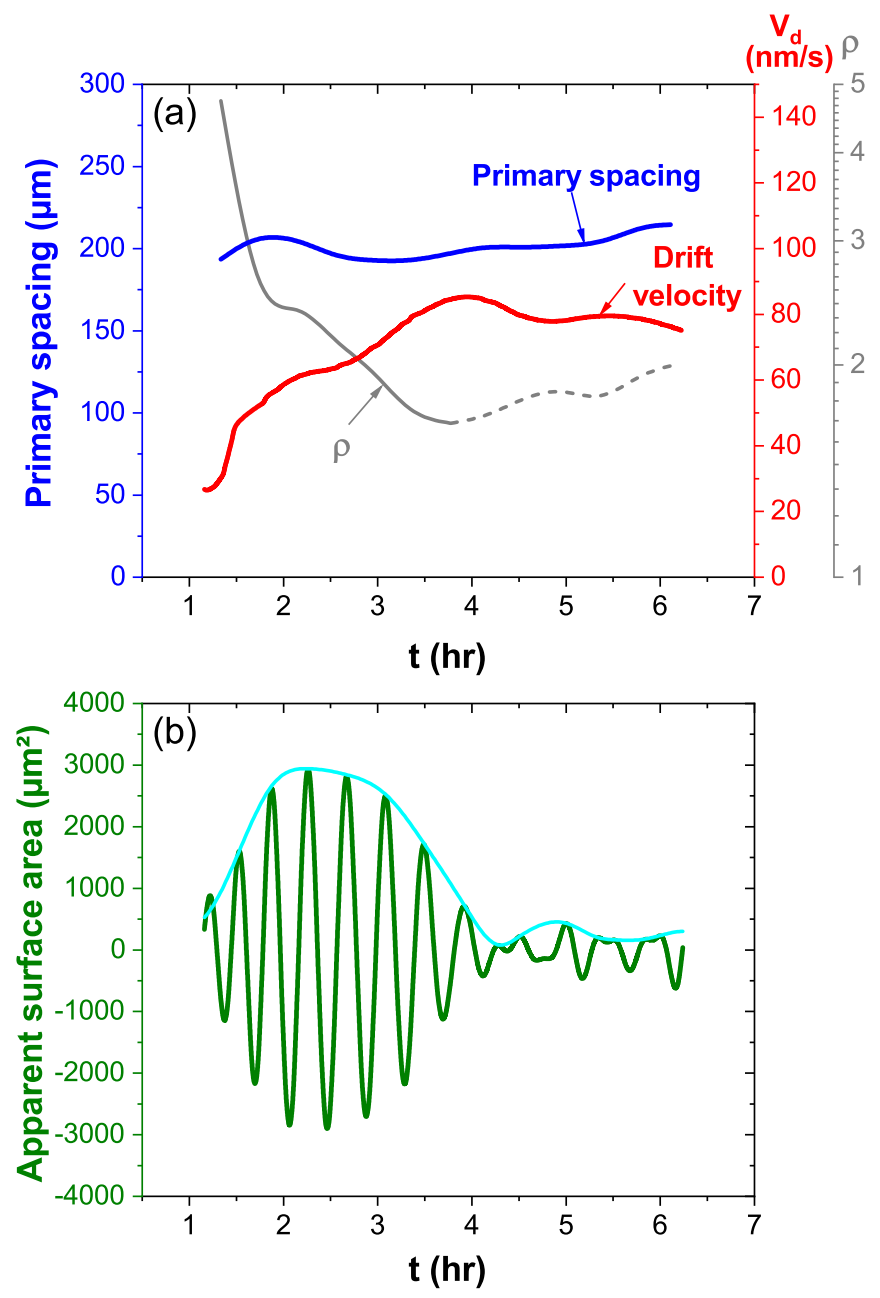

FIG. 4. Typical time evolution of a cell, belonging to the left side of the interface (stops oscillating), characterized by (a) its primary spacing (blue curve), drift velocity (red curve), and (b) its apparent surface area (green curve). The gray curve in (a) is the evolution of the ratio $\rho$ between the characteristic times of drift and oscillation. The cyan (upper) curve in (b) corresponds to the upper envelope of the apparent surface area. $\left(V_{p}=1.5 \mu \mathrm{m} / \mathrm{s} ; G=19 \mathrm{~K} / \mathrm{cm}\right)$.

The growth angle $\theta_{g}$ varies relatively to $\theta_{0}$ as

$$
\theta_{g} / \theta_{0}=1-\frac{1}{1+f \mathrm{Pe}^{g}}
$$

where $f$ and $g$ are alloy-dependent constants, and the Péclet number $\mathrm{Pe}=\left(\lambda V_{p}\right) / D$, with $\lambda$ the primary spacing and $D$ the solute diffusion coefficient [15-17,23]. Phase-field simulations of perfectly hexagonal patterns with different angles $\theta_{0}$ lead to $f=0.67$ and $g=1.47$ (unpublished work). The time evolution of the average primary spacing is presented in Figs. 3(b) and 3(d) for the two selected regions: the morphological difference also affects the primary spacing since the right region presents a primary spacing roughly $11 \%$ higher than the left region for $V_{p}=1.5 \mu \mathrm{m} / \mathrm{s}$, and $4.5 \%$ higher for $V_{p}=0.75 \mu \mathrm{m} / \mathrm{s}$. Based on the measurement of the average primary spacing and the corresponding average drift velocity, the misorientation angles $\theta_{0}$ are estimated for the right and left selected regions of the interface (Table II). It should be

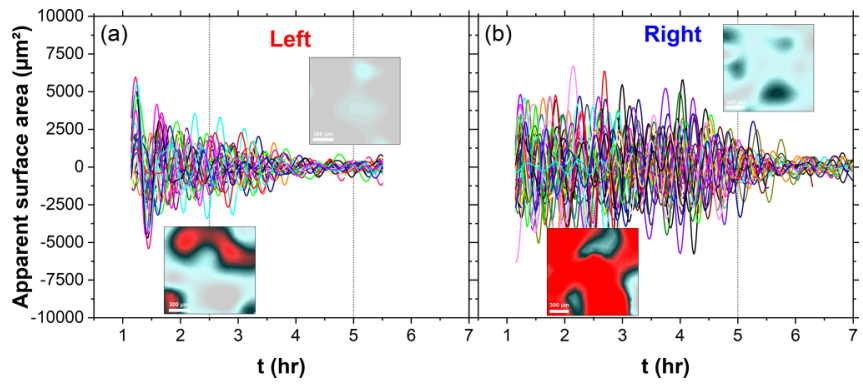

FIG. 5. Evolution of the apparent surface area for the cells present in the (a) left and (b) right sides of the interface, for $V_{p}=$ $1.5 \mu \mathrm{m} / \mathrm{s}$ and $G=19 \mathrm{~K} / \mathrm{cm}$. The insets represent colormaps of the oscillation amplitude at the times given by the gray dotted lines, where the light gray color represent $A$ lower than $300 \mu \mathrm{m}^{2}$ and red (darker in the grayscale version) greater than $2500 \mu \mathrm{m}^{2}$.

mentioned that for $V_{p}=0.75 \mu \mathrm{m} / \mathrm{s}$ a stationary-state was not attained on either primary spacing or in drift velocity. This is probably related to the fact that at $V_{p}=0.75 \mu \mathrm{m} / \mathrm{s}$, the interface is macroscopically convex. This curvature induces a glide of cells along the interface slope from the center towards the border, and the primary spacing evolution results from a permanent balance between the cell stretching at the center and the elimination at the border. The values at the end of the solidification were used to estimate the misorientation angles $\theta_{0}$.

\section{Oscillation characterization}

In top-view images, cells appear bright due to the light guided through their body and the size of the bright area is defined as the apparent surface area. The oscillation analyses are based on the time evolution of the apparent cell area [8,22]. For each of the cells previously identified inside the red (white) and blue (black) square zones of Fig. 1, the apparent surface area was followed as a function of the solidification time. An example of the typical behavior of a cell that stops oscillating is given in Fig. 4. The primary spacing presents a small overshoot at the end of the coarsening stage and then progressively increases till the end of the solidification. The drift velocity increases to reach a stationary value after $4.5 \mathrm{~h}$ roughly. The oscillation amplitude increases to reach its maximum after $2 \mathrm{~h}$, it starts decreasing significantly after $3.3 \mathrm{~h}$ and oscillations almost stop after $4.2 \mathrm{~h}$.

The evolution curves of all the studied cells in the different areas of interest are superposed in Fig. 5. As seen in Table II, the oscillation period $\tau_{\text {osc }}$ on the right side of the interface (blue - or black-square) is slightly longer than the one on the left side (red-or white—square) for both values of $V_{p}$. The oscillation amplitude is much more important and stable on the right than on the left side, where it seems to progressively decrease, as evidenced by the colormap insets in Fig. 5 for $V_{p}=1.5 \mu \mathrm{m} / \mathrm{s}$. From Fig. 5 , it is difficult to determine the exact moment when oscillations stop. To clarify this point, the upper envelope of each curve [for example, the cyan-upper-curve in Fig. 4(b)] is extracted. At time $t$, the envelope amplitudes are averaged over all the cells, giving a curve $A_{\text {av }}(t)$. Dividing $A_{\text {av }}(t)$ by the curve maximum, one obtains $\tilde{A}_{\mathrm{av}}(t)$ that oscillates between 0 and +1 , as shown 

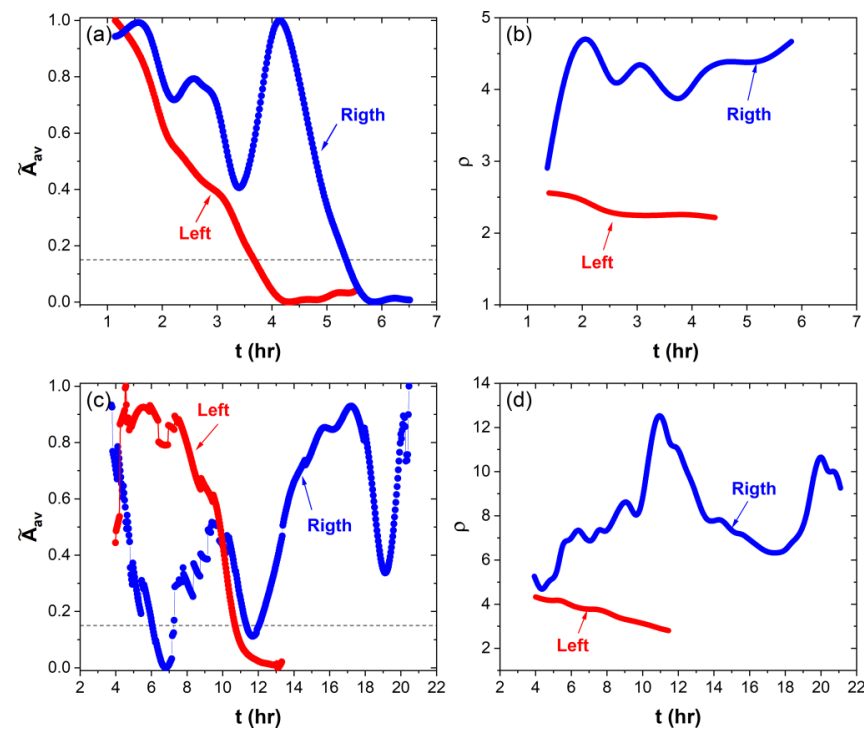

FIG. 6. Evolution of (a), (c) the apparent surface area and (b), (d) the criterion parameter $\rho=\tau_{\text {drift }} / \tau_{\text {osc }}$ for the cells present in the left and right sides of the interface: $G=19 \mathrm{~K} / \mathrm{cm} ; V_{p}=1.5 \mu \mathrm{m} / \mathrm{s}$ (upper panels) and $V_{p}=0.75 \mu \mathrm{m} / \mathrm{s}$ (lower panels).

in Figs. 6(a) and 6(c). A great difference can be seen in the mean amplitude curves obtained on both sides of the interface: on the left side, $\tilde{A}_{\mathrm{av}}(t)$ decreases smoothly to reach a steady value for both pulling rates. On the right side, the oscillation dynamics depends on the pulling velocity: for $V_{p}=1.5 \mu \mathrm{m} / \mathrm{s}$, the amplitude remains significant, albeit quite variable, before starting to decrease after $4 \mathrm{~h}$ to reach its minimum value at $5.8 \mathrm{~h}$; for $V_{p}=0.75 \mu \mathrm{m} / \mathrm{s}$, the amplitude remains high. Based on the error associated with the apparent surface area measurement, we define a cutoff of $300 \mu \mathrm{m}^{2}$ (gray dashed line) below which we consider that there is no oscillation. This roughly corresponds to the condition $\tilde{A}_{\mathrm{av}}(t) \leqslant 0.15$. From the oscillation curves of Fig. 6 , we estimated the time $t_{\text {stop }}$ when oscillations stop according to this cutoff (Table II). In our previous work [8], we came to the conclusion that the drift induced by growth misorientation inhibits oscillation when a threshold of drift velocity is reached. Drift and oscillation have their own dynamics, but the oscillation is based on a subtle evolution of the concentration field at the tip of the cell and adding a drift could affect the dynamics of oscillation by modification of this concentration field, and eventually inhibit it if the drift is too important. We defined a criterion based

TABLE II. Pattern and oscillation characteristics measured in experiments. s.d. corresponds to the standard deviation of $\tau_{\mathrm{osc}}$.

\begin{tabular}{lrrrrr}
\hline \hline$V_{p}(\mu \mathrm{m} / \mathrm{s})$ & \multicolumn{3}{c}{1.5} & & \multicolumn{2}{c}{0.75} \\
\cline { 2 - 3 } \cline { 5 - 6 } Interface region & Left & Right & & Left & Right \\
\hline$\theta_{0}(\mathrm{deg})$ & 6.5 & 3.1 & 6.7 & 2.5 \\
$\tau_{\text {osc }}$ & 22.1 & 27.0 & & 64.1 & 77.1 \\
\pm s.d. $(\mathrm{min})$ & 1.6 & 2.0 & & 9.5 & 8.7 \\
$t_{\text {stop }}(\mathrm{h})$ & 4.1 & 5.8 & & 11.3 & - \\
$\rho_{\text {stop }}$ & 2.3 & N.A. & & 2.8 & - \\
\hline \hline
\end{tabular}

on the ratio of the characteristic times of oscillation and drift, estimated, respectively, as the oscillation period and the time necessary for a cell to glide over its width (ratio between the primary spacing and the drift velocity),

$$
\rho=\frac{\tau_{\text {drift }}}{\tau_{\text {osc }}} .
$$

Our previous analyses [8] based on the observation of the experiments at $V_{p}=0.75$ and $1.5 \mu \mathrm{m} / \mathrm{s}$ led us to propose a possible threshold $\rho_{\text {stop }}=3.0$ below which the oscillation is inhibited. The evolution of this ratio for a cell is shown in Fig. 4 (gray curve): at some point roughly corresponding to a threshold $\rho_{\text {stop }}=1.7$, the cell is no longer oscillating. From that point on, the gray curve turned into dashed because $\rho$ is estimated using the oscillation period measured before the oscillation stop. The time evolution of the average value of $\rho$ is represented in Figs. 6(b) and 6(d) for each region. On the left side of the interface, for both pulling rates, the curves start with a quite low value and constantly decrease with the oscillation amplitude. The average values of $\rho_{\text {stop }}$ corresponding to the moment when the oscillations stop are given in Table II. We should point out that in the previous work, the values were estimated using the mean values of primary spacing and drift velocity (Table II of Ref. [8]), while in this work $\rho$ is calculated for each cell (using its own local primary spacing and drift velocity) at each time and is therefore more precisely determined [Fig. 6(b)]. On the right side of the interface, the parameter $\rho$ is always higher than its value on the left side, thus justifying a more robust oscillations. For $V_{p}=1.5 \mu \mathrm{m} / \mathrm{s}$, the oscillation stops after roughly $6 \mathrm{~h}$, even if the parameter $\rho$ is high. However, as was demonstrated experimentally and numerically in [8], the range of experimental parameters over which the oscillation can develop is limited. Especially, in our case, oscillation occurs for a velocity range of $2<V_{p} / V_{c}<6\left(V_{c}\right.$ being the critical velocity for planar interface destabilization). Close to the upper limit of this velocity range, oscillations occur only during the transient growth stage. The oscillation stops on the right side of the interface for $V_{p}=1.5 \mu \mathrm{m} / \mathrm{s}$ because the interface velocity attains the upper limit of velocity range to get oscillations. This is why in Table II we consider $\rho_{\text {stop }}$ as nonapplicable (N.A.).

\section{PHASE-FIELD}

Two decades ago, Alain Karma and coworkers introduced the thin interface phase-field model (TIPM) to simulate the solidification of a pure substance [24,25]. This model was extended later to the case of a dilute binary alloy [26,27]. Since, many examples of 3D simulations of alloy solidification that give quantitative agreement with the experiments were reported [5-8,15,28,29], following K. Glasner, we replace the usual phase-field $\varphi \in[-1,1]$ by the preconditioned phase-field

$$
\psi=\sqrt{2} \tanh ^{-1}(\varphi),
$$

which is a signed distance from the interface, $\psi \in$ $(-\infty,+\infty)$ [30]. This transformation significantly reduces the numerical effort because a larger nondimensional mesh size can be used without affecting the numerical accu- 
racy [30]. The code is made even faster by using graphics processing unit (GPU) parallel programming [6-8,31,32]. We now recall the main lines of the model. More details can be found elsewhere $[5,15]$.

\section{A. Evolution equations}

In the TIPM, the solid-liquid interface has a finite width $W_{0}[24,25]$. For rough materials like succinonitrile, the kinetic coefficient can be safely set to zero. To ensure this, the time unit must verify the condition $\tau_{0}=a_{0} W_{0}^{3} /\left(D d_{0}\right)$, where $d_{0}$ is the capillary length, $D$ the solute diffusion constant in the liquid phase, and $a_{0} \simeq 0.5539$ [24,25]. In the following, lengths and times are, respectively, divided by $W_{0}$ and $\tau_{0}$, which leads to the following nondimensional evolution equation for $\psi$ :

$$
\begin{aligned}
\left(1-\beta_{k} z^{*}\right) a_{s}^{2} \frac{\partial \psi}{\partial t}= & \sqrt{2}\left[\varphi-C\left(1-\varphi^{2}\right)\left(U+z^{*}\right)\right] \\
& +a_{s}^{2}\left[\nabla^{2} \psi-\sqrt{2} \varphi(\vec{\nabla} \psi)^{2}\right] \\
& +2 a_{s} \vec{\nabla} a_{s} \vec{\nabla} \psi+\frac{\sqrt{2}}{\left(1-\varphi^{2}\right)} \vec{\nabla} \vec{A},
\end{aligned}
$$

with $\beta_{k}=(1-k), k$ being the partition coefficient. Here, the nondimensional concentration field $U$ is related to the physical concentration field $c$ by

$$
c=\frac{c_{\infty}}{k}\left(1+\beta_{k} U\right)\left(\frac{\alpha_{k}-\beta_{k} \varphi}{2}\right),
$$

where $\alpha_{k}=(1+k)$, and the additional term $\beta_{k} z^{*}$ on the lefthand side of Eq. (5) is introduced to reduce higher order corrections that appear in the asymptotic expansion of the phase-field equations [27]. The nondimensional variable $z^{*}$ is defined as

$$
z^{*}=\left[z+z_{s}(t)-V t\right] / l_{T},
$$

where

$$
l_{T}=m c_{\infty}(k-1) /(k G)
$$

is the thermal length. The quantity $V t-z_{s}$ is a small vertical offset that becomes constant (up to one mesh size) at large times [5]. For our rough material, the constant that couples the nondimensional concentration field $U$ to the phase-field is $C=75 D^{*} / 47$, with $D^{*}=D \tau_{0} / W_{0}^{2}$ [25].

In Eq. (5), both $a_{s}$ and $\vec{A}$ depend on the crystal anisotropy. In the present study we consider the situation where the crystal axes $X, Y, Z$ are rotated with respect to the axes $x, y, z$ of the computational domain that has the shape of a rectangular parallelepiped. The vertical axis $z$ gives the direction of the imposed temperature gradient, $x$ is the coordinate along the domain width and $y$ lies along the domain thickness. Three rotation angles are needed in general, but we consider here a simple rotation of the [100] and [001] crystal axes about the $y=Y=[010]$ crystal axis, by an angle $\theta_{0}$. In the $X, Y, Z$ reference frame, the anisotropy function has the usual form for cubic crystals [33],

$$
a_{s}=\left(1-3 \epsilon_{4}\right)+4 \epsilon_{4}\left(n_{X}^{4}+n_{Y}^{4}+n_{Z}^{4}\right),
$$

where $n_{X}, n_{Y}, n_{Z}$ are the components of unit vector $\vec{n}$ and $\epsilon_{4}$ is the anisotropy strength. Then, the three components of the anisotropy vector $\vec{A}$ are

$$
A_{v}=16 \epsilon_{4} \frac{\left(1-\varphi^{2}\right)}{\sqrt{2}}|\vec{\nabla} \psi| a_{s} n_{v}\left[\left(n_{X}^{4}+n_{Y}^{4}+n_{Z}^{4}\right)-n_{\nu}^{2}\right],
$$

where $v=X, Y, Z$. The numerical calculations are actually performed in the domain reference frame, $x, y, z$, and they make use of the very simple transformation equations between the two frames of reference:

$$
\begin{aligned}
& n_{X}=\sin \theta_{0} n_{x}+\cos \theta_{0} n_{z}, \\
& n_{Y}=n_{y}, \\
& n_{Z}=\cos \theta_{0} n_{x}-\sin \theta_{0} n_{z} .
\end{aligned}
$$

We use the one-sided model that neglects solute diffusion in the solid. Following Refs. [26,27], a corrective solute current

$$
\vec{j}_{a t}=\frac{W_{0}}{2 \sqrt{2}} \frac{c_{\infty}}{k} \beta_{k} \gamma \vec{n},
$$

with $c_{\infty}$ the nominal solute concentration, is used to avoid artificial solute trapping and other spurious corrections due to the finite interface thickness $W_{0}$. Here,

$$
\gamma=\frac{1-\varphi^{2}}{\sqrt{2}}\left(1+\beta_{k} U\right) \frac{\partial \psi}{\partial t} .
$$

The evolution equation for the nondimensional concentration field $U$ is then $[26,27]$

$$
\begin{aligned}
\left(\alpha_{k}-\beta_{k} \varphi\right) \frac{\partial U}{\partial t}= & (1-\varphi) D^{*} \nabla^{2} U \\
& -\frac{\left(1-\varphi^{2}\right)}{\sqrt{2}} D^{*} \vec{\nabla} \psi \vec{\nabla} U \\
& -\frac{1}{\sqrt{2}} \vec{n} \vec{\nabla} \gamma+\gamma\left(1-\frac{\vec{\nabla} \vec{n}}{\sqrt{2}}\right) .
\end{aligned}
$$

\section{B. Parameters and conditions imposed}

Along the $y$ direction (sample thickness), no-flux boundary conditions are imposed on $U$ and $\psi$. Periodic boundary conditions are imposed on $U$ and $\psi$ in the $x$ direction (sample width) because the cellular pattern is expected to constantly drift due to the crystal misorientation. Along $z$, the boundary conditions are no-flux at the bottom of the domain and $U=-1.0$ at its top. In addition, the fields $\psi$ and $U$ are shifted when needed to keep the interface roughly at the same vertical location in the simulation domain.

The initial condition is a flat interface that is perturbed by a random spatiotemporal noise of weak amplitude $[6-8,15,34,35]$ for a moderate time $(1000 \mathrm{~s}$ in most simulations). Beyond that time, the spatiotemporal noise is turned off. To avoid numerical divergences due to the very fast destabilization of the flat interface, simulations usually start with a rather high value of the temperature gradient (typically $G=80 \mathrm{~K} / \mathrm{cm}$ ). This value is lowered to reach the nominal imposed value $(G=30 \mathrm{~K} / \mathrm{cm})$ in about $1000 \mathrm{~s}$. The numerical interface parameter $\xi=W_{0} / d_{0}$ is taken sufficiently small that good convergence of the simulation results with diminishing interface width is ensured [5,15]. 
TABLE III. Control and numerical parameters used in the simulations.

\begin{tabular}{lccc}
\hline \hline Control parameter & Symbol & Magnitude & Unit \\
\hline Thermal gradient & $G$ & 30 & $\mathrm{~K} / \mathrm{cm}$ \\
Pulling velocity & $V_{p}$ & 1.5 & $\mu \mathrm{m} / \mathrm{s}$ \\
\hline Numerical parameter & & Expression & Value \\
\hline Convergence & & $W_{0} / d_{0}$ & 160.0 \\
Grid spacing & & $\delta s / W_{0}$ & 1.2 \\
\hline \hline
\end{tabular}

The control and numerical parameters used in the simulations are gathered in Table III. The values of $G$ and $V_{p}$ reported in this table should not be directly associated with the corresponding values of the experimental control parameters (Table I). Indeed, in recent studies, it has been repeatedly observed that a systematic shift between the numerical and experimental conditions must be made to obtain comparable dynamical behaviors $[7,8]$.

To scan the growth dynamics as a function of the [001] crystal axis inclination, the misorientation angle $\theta_{0}$ is varied in the simulations, according to a predefined linear ramp. A typical time evolution imposed on $\theta_{0}$ is represented in Fig. 7. Misorientation is initially set to zero for a time (8000 s) sufficient for the system to leave the transient regime, then $\theta_{0}$ is increased linearly until a time (28000 s) when a prescribed maximum inclination $(10 \mathrm{deg})$ is reached. After that, $\theta_{0}$ is decreased symmetrically and, when zero inclination is reached again (48000 s), a more or less long time is still simulated. As seen in Fig. 7, the variations of $\theta_{0}$ are taken sufficiently slow that the cell drift velocity follows them without noticeable delay. Small differences that appear can be mainly attributed to numerical approximations made to extract the cell drift velocity from an upper view of the system and also to the

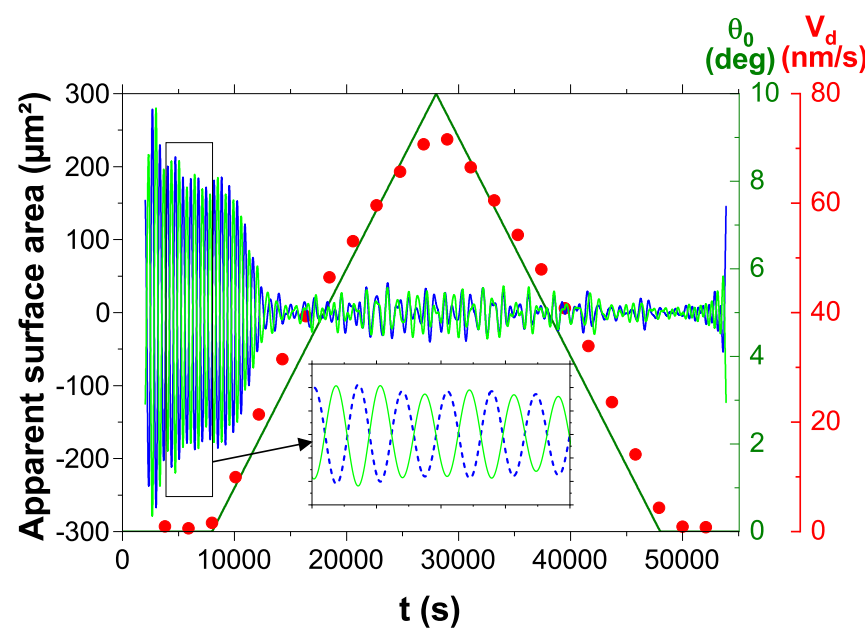

FIG. 7. Transition from the $2 \lambda-\mathrm{O}$ oscillatory to a nonoscillatory mode in the narrow thin sample. Time evolution of the inclination angle $\theta_{0}$ (straight lines), of the average drift velocity $V_{d}$ (symbols), and of the apparent surface areas of the two cells (curves). The inset is a closer view of the latter in the region delimited by the rectangular on the left side of the figure.
TABLE IV. Domain size and oscillation characteristics measured in the numerical simulations without spatiotemporal noise. Errors on $\tau_{\text {osc }}$ are about $1.0 \mathrm{~min}$.

\begin{tabular}{lccc}
\hline \hline Sample & Thin narrow & Thin wide & Bulk \\
\hline Section area $\left(\mu \mathrm{m}^{2}\right)$ & $240.0 \times 60.0$ & $1143.1 \times 60.0$ & $748.7 \times 748.7$ \\
$\tau_{\text {osc }}(\min )$ & 12.0 & 14.0 & 13.0 \\
$\theta_{0, \text { stop }}(\mathrm{deg})$ & 2.2 & 8.1 & 15.1 \\
$t_{\text {stop }}(\mathrm{h})$ & 3.45 & 6.75 & 6.45 \\
$\rho_{\text {stop }}$ & 7.7 & 1.9 & 1.4 \\
\hline \hline
\end{tabular}

small number of cells involved here (two). The agreement further improves in large bulk samples.

\section{Numerical simulations in thin samples}

As mentioned previously, the present simulation code is based on earlier numerical studies of directional solidification of an acrylonitrile-succinonitrile alloy in thin samples $[5,15]$. To make contact with the microgravity experiments considered here, we now replace this alloy with the camphor-succinonitrile alloy that is used in DECLIC and we first perform numerical simulations in the thin sample geometry. Experimental estimates of camphor-succinonitrile physical parameters are listed in Table I. The control and numerical parameters used in the simulations are those of Table III. The numerical domain is approximately $2143.3 \mu \mathrm{m}$ in height ( $z$ axis) and $60.0 \mu \mathrm{m}$ in thickness $(y$ axis). Two widths ( $x$ axis) are considered: $240.0 \mu \mathrm{m}$ for the narrow domain that contains a pair of cells and $1143.1 \mu \mathrm{m}$ for the wide domain that contains about ten cells. The main oscillation characteristics estimated from the numerical data described in this section and the next one are gathered in Table IV.

We first consider simulations in the narrow system. Two cells form and oscillate in phase opposition $(2 \lambda-\mathrm{O}$ mode $)$ with a period $\tau_{\mathrm{osc}}=12 \pm 1 \mathrm{~min}$ (see Table IV), a value very close to that found previously for $2 \pi / 3$ oscillations for the same parameters [8]. The small sensitivity of the oscillation period to the actual oscillation mode was already noted previously, and it is confirmed here for the $2 \lambda-\mathrm{O}$ mode. For this small system, the existence of a well-defined static threshold between oscillatory and nonoscillatory states can be addressed, provided that only two cells coexist and fixed misorientations $\theta_{0}$ are considered. As we will see later, there is no simple answer to this question when many cells coexist because oscillations are strongly affected by local rearrangements that constantly occur in the cellular array. As seen here and in the next paragraph, differences in the initial conditions (shape and/or imposed noise) and in the inclination (fixed versus variable) may also result in different thresholds for the present thin system. To locate the static threshold, we impose a constant misorientation $\theta_{0}$ and we start with a thin domain of width slightly smaller than half that of the final domain. A single inclined cell with a steady growth velocity is then obtained. This cell is slightly dilated and copied twice in the final domain that has the necessary width for the $2 \lambda-O$ mode to exist $(240.0 \mu \mathrm{m})$. Growth is resumed from there, with a small spatiotemporal noise imposed during a short initial time length (100 s). Figure 8 shows the obtained oscillation 


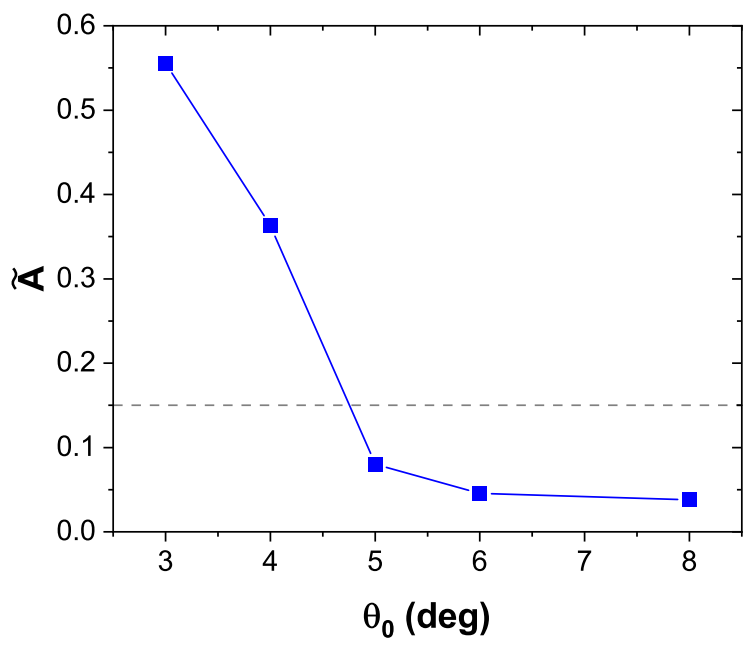

FIG. 8. Evolution of the oscillation amplitude as a function of fixed inclination angles $\theta_{0}$ in a thin narrow domain.

amplitudes $\tilde{A}$ as a function of fixed misorientation angles $\theta_{0}$. Clearly, $\tilde{A}$ decreases below the cutoff experimental amplitude 0.15 for a static threshold $\theta_{0 \text {,static }} \simeq 4.5 \mathrm{deg}$. This result compares well with but is not equal to the dynamic thresholds $\theta_{0 \text { stop }}$, as we see now.

We now turn to the simulations with a variable misorientation, as described at the end of Sec. III B. The oscillations survive after the misorientation has started to increase, until a value $\theta_{0 \text {,stop }} \simeq 2.2 \mathrm{deg}$ is reached; at higher misorientations, the cells do not oscillate any more. Figure 9(a) represents the time evolution of $\theta_{0}, \rho$, and $\tilde{A}_{\mathrm{av}}$. Using the experimental cutoff for oscillation detection, $\tilde{A}_{\text {av }}=0.15$, one sees that oscillations stop for $\rho_{\text {stop }} \simeq 7.7$. The oscillations do not resume during the decrease of $\theta_{0}$, as could be expected. This is rather predictable because there is little possibility of size fluctuations in such a small system and because the noise is turned down at that time. However, keeping the spatiotemporal noise during the whole simulation shows that oscillations resume roughly for $\rho_{\text {start }} \simeq \rho_{\text {stop }}$ when $\theta_{0}$ is decreased [see Fig. 9(b)]. The amplitude of the noise imposed on the phase-field $\psi$ at each time step is $F_{0}=5.0 \times 10^{-4}$. We observe then more irregular variations of $\tilde{A}_{\mathrm{av}}(t)$, and obtain a lower (but less accurate) estimate of the oscillatory-non oscillatory threshold, $\rho_{\text {stop }} \simeq 5.2$, while $\theta_{0, \text { stop }} \simeq 3.8 \mathrm{deg}$ which is closer to $\theta_{0, \text { static }}$. Thus, the imposed spatiotemporal noise induces sufficient disorder in the system to trigger oscillations. From now on, we will consider larger systems that contain tens of cells. In these large systems, the size and local environment varies from cell to cell. Due to this geometrical disorder, oscillations start and stop spontaneously, and so, except for the initial phase, spatiotemporal noise is set to zero in the following.

We now turn to the wide system that consists of about ten cells arranged in a thin layer [Fig. 10(a)]. Figure 10(b) represents the time evolution of $\theta_{0}, \rho$, and $\tilde{A}_{\text {av }}$. The estimated oscillation period is $\tau_{\mathrm{osc}}=14 \pm 1 \mathrm{~min}$ for this simulation (see Table IV). As just discussed, oscillations are more likely to start and stop, as compared to the narrow system, because cell spacings and shapes now vary significantly in time and space. For this reason, one sees that oscillations resume at late
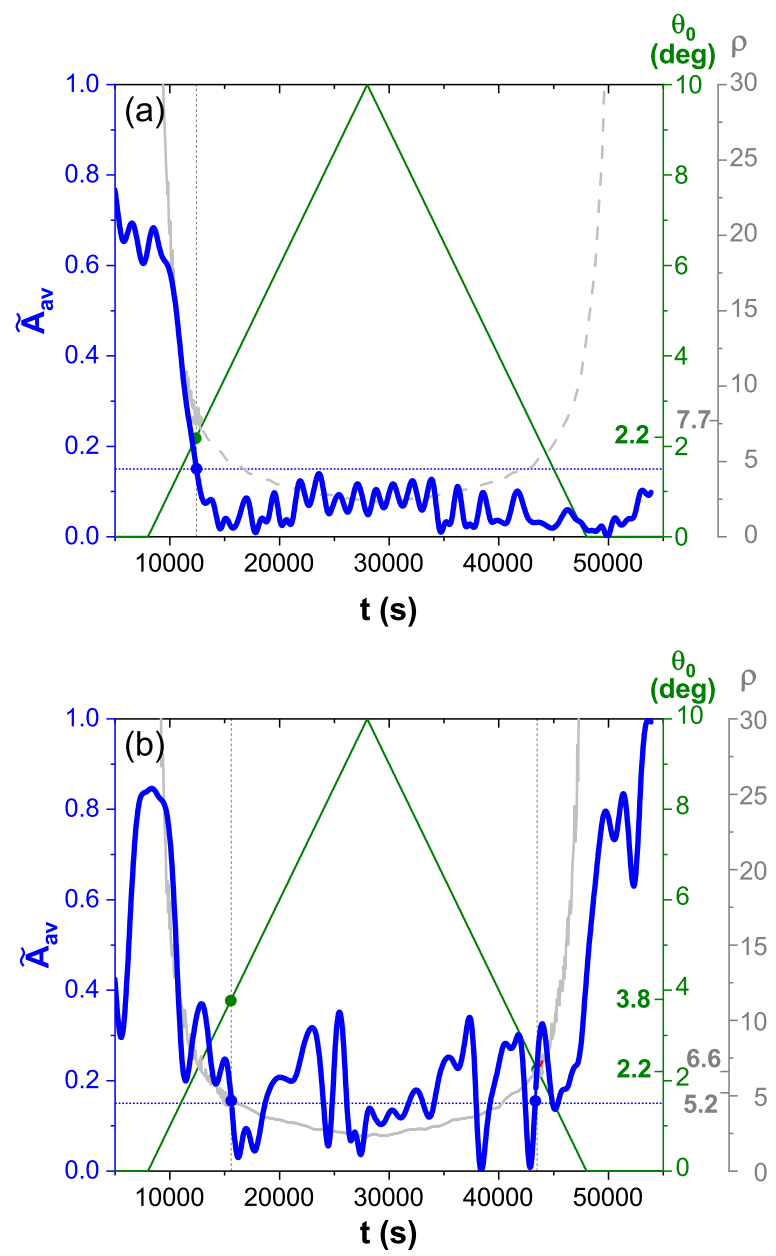

FIG. 9. Time evolution of the oscillation characteristics obtained by numerical simulations in a thin narrow domain: (a) without imposing spatiotemporal noise and (b) imposing a spatiotemporal noise of amplitude $F_{0}=5.0 \times 10^{-4}$. Normalized average apparent surface area $\tilde{A}_{\mathrm{av}}$, thicker and darker curve; Inclinaiton angle $\theta_{0}$, straight line; Ratio of characteristic times of oscillation and drift $\rho$, light gray curve.

times, far on the right side of the figure. Oscillations do not reappear earlier because no spatiotemporal noise is imposed and because the confinement due to the very small sample thickness does not allow much rearrangement of the cells in the transverse direction. A much stronger effect indeed results from the local ordering of neighbor cells in the bulk samples studied in DECLIC experiments. To take local ordering of the cellular array into account in the phase-field numerical simulations, we now consider the cellular pattern evolution in extended domains.

\section{Numerical simulations in bulk samples}

Simulations in bulk systems are performed in domains with a square $x y$ cross section of area $748.7 \times 748.7 \mu \mathrm{m}^{2}$ and a depth $z$ of $2143.3 \mu \mathrm{m}$. Since local ordering is expected to fluctuate in time and space, with noticeable repercussions on the solidification dynamics, we opt to perform a series of 4 simulations that differ only by their initial condition (a different seed for the random number generator). The amplitude 
(a)
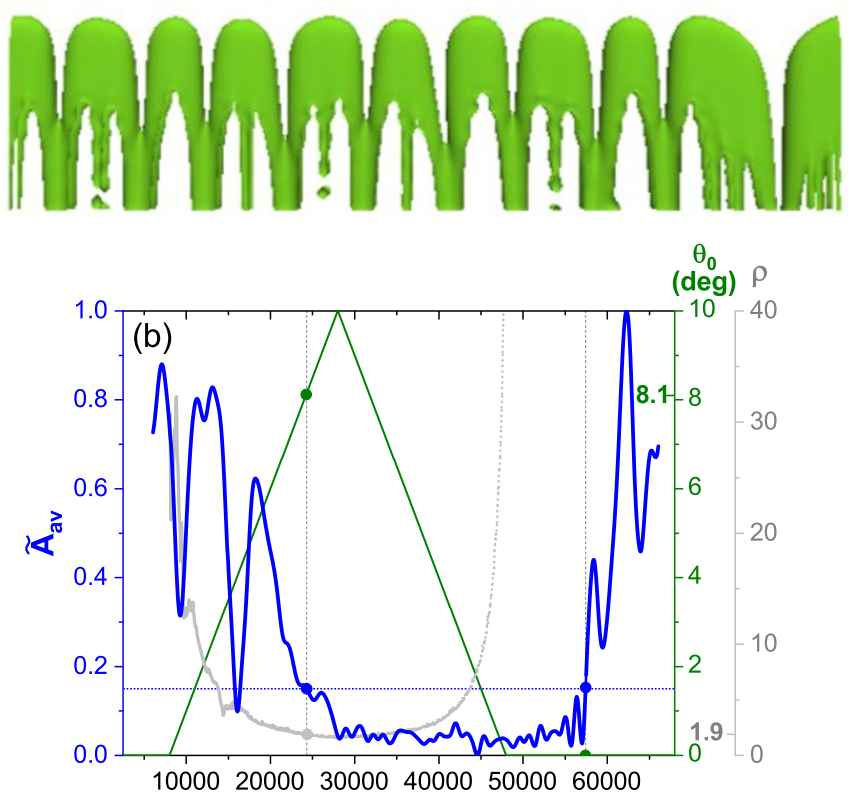

t (s)

FIG. 10. Numerical simulations in a thin wide domain. (a) Snapshot of the system showing the solidification cells ( $\psi=0$ contour) at time $t=57000 \mathrm{~s}$ when oscillations start again. (b) Time evolution of the oscillation characteristics. Normalized average apparent surface area $\tilde{A}_{\mathrm{av}}$, thicker and darker curve; inclinaiton angle $\theta_{0}$, straight line; ratio of characteristic times of oscillation and drift $\rho$, light gray curve.

curves of all the cells in the domain are displayed in Fig. 11 for these four different simulations.

As expected, the oscillations in the four simulations are different but all the curves share the common trend for oscillatory states to disappear at some point during the crystal misorientation increase and to reappear at some point during its decrease. In the central, nonoscillatory domain, bursts of a few oscillating cells appear at times. One will see later that

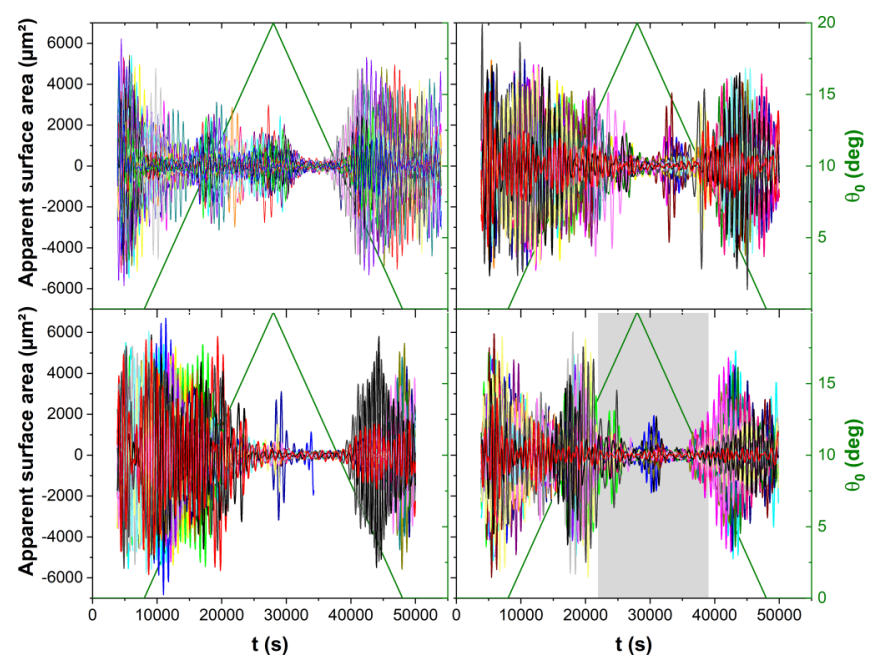

FIG. 11. Time evolution of the cell apparent surface area in four independent simulations of a bulk system.

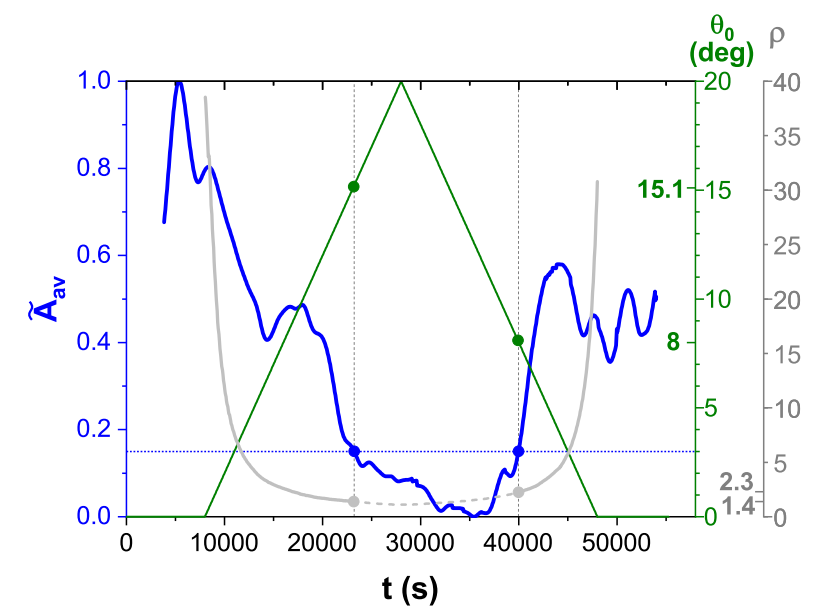

FIG. 12. Time evolution of the oscillation characteristics averaged over four independent numerical simulations in a bulk domain. Normalized average apparent surface area $\tilde{A}_{\mathrm{av}}$, thicker and darker curve; inclination angle $\theta_{0}$, straight line; ratio of characteristic times of oscillation and drift $\rho$, light gray curve.

bursts result from local interactions within small groups of a few neighboring cells. The four amplitude curves of Fig. 11 are summed up and the resulting amplitude is again divided by its maximum value to get $\tilde{A}_{\text {av }}$. A similar average is made to obtain the oscillation parameter $\rho$. The curves giving $\tilde{A}_{\mathrm{av}}(t)$ and $\rho(t)$ are displayed in Fig. 12, together with the curve $\theta_{0}(t)$. It should be noted that the highest misorientation explored in this series of simulations is 20 degrees (instead of 10 degrees in the thin sample simulations) because the larger fluctuations in the cellular array that result from an increase of the degrees of freedom in the sample thickness make it less likely for a given cell to stop oscillating. From the curves displayed in Fig. 12, one obtains the estimate $\rho_{\text {stop }}=1.4$ for the oscillatory-nonoscillatory transition threshold. This result quantitatively agrees with the one predicted by the analysis of our experimental data (see Sec. II C).

In these bulk sample simulations, we obtain an oscillation period $\tau_{\mathrm{osc}}=13 \pm 1 \mathrm{~min}$. Within the error bars, this estimate is compatible with the ones found above in thin systems (both narrow and wide) and, as already mentioned, is also compatible with the estimate obtained previously for a well ordered hexagonal array of oscillating cells [8]. This suggests that all the cells basically behave as oscillators with identical natural periods but that global and local geometrical constraints influence their actual oscillatory state via short range couplings that slightly modify the oscillation periods from one cell to another. Quantifying this picture would further require a close analysis of the concentration field that mediates the cell-to-cell interactions resulting from a subtle feedback between individual cell shapes, local ordering of the cellular array, and local solute fluxes. Such a complete analysis was recently proposed in the case of the $2 \lambda-\mathrm{O}$ mode in thin samples [5] but we will not attempt to generalize it to the more complex geometries encountered here.

To investigate the origin of the previously mentioned oscillation bursts that occur during the nonoscillatory (high misorientation) regime, we found that the cells presenting 

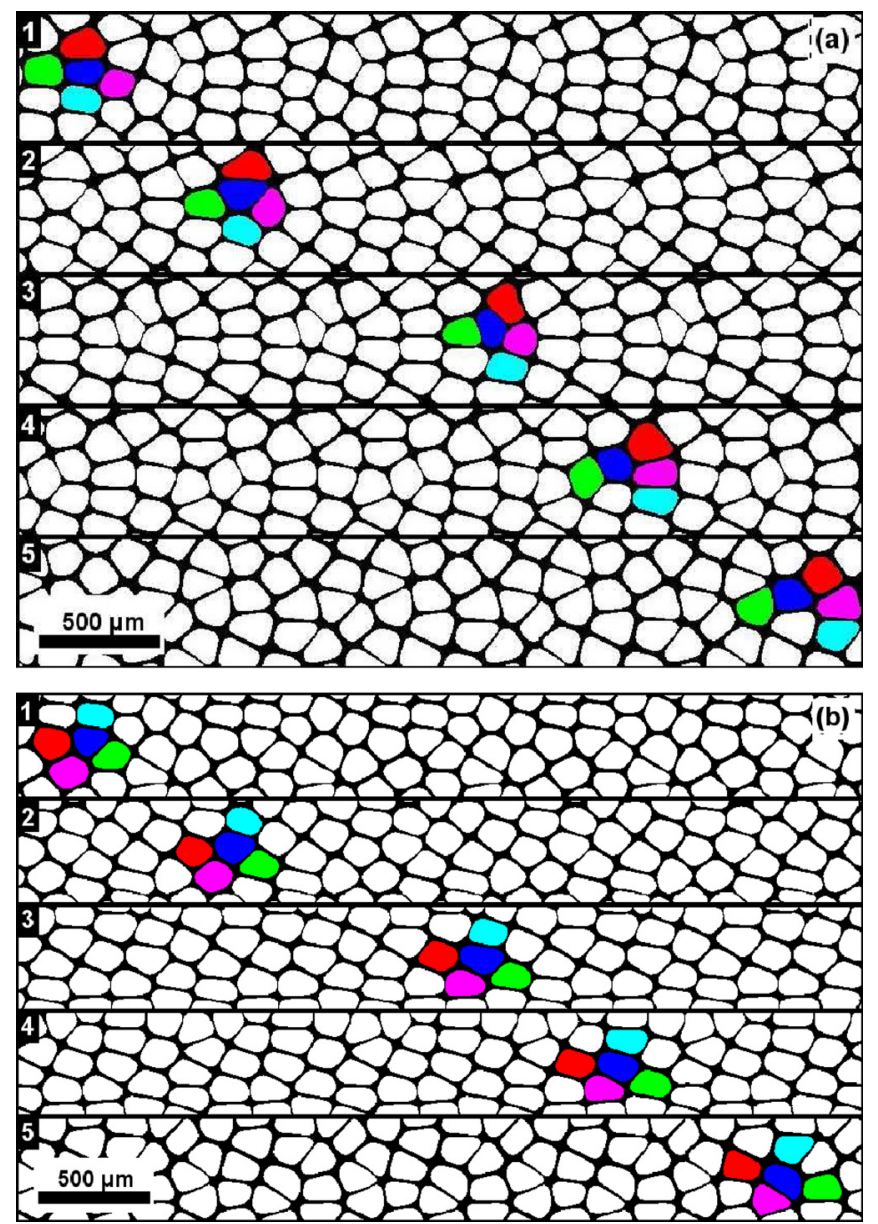

FIG. 13. Temporal sequence showing the evolution of a local group of 5 cells: (a) unstable group and (b) stable group. Both cell groups relate to the lower right panel of Fig. 11, where the time interval considered is shaded in gray. this behavior are first neighbors and we focused on the time evolution of a group of five cells [Fig. 13(a)]. The spatial organization of this group, termed unstable, changes in time: two cells that were initially not in contact with the central cell have drifted faster and pushed their way to become part of the group at the end of the sequence. As a consequence, several cells have started rather strong oscillations as evidenced by the time evolution of the normalized cell surface areas $\tilde{A}(t)$ [Fig. 14(a)]. The three highest oscillation amplitudes (green, blue, red cells) roughly occur when the two extra white cells make contact with the group.

In the close neighborhood of the unstable group, we found another group of five cells, termed stable, which keeps roughly its initial spatial organization throughout the time sequence [Fig. 13(b)]. As a result, the influence of the second neighbor cells remains rather low and the cells of the group do not start oscillating [Fig. 14(b)]. All the curves remain below the cutoff value of 0.15 , except for one that slightly increases beyond 0.15 at intermediate times (cyan curve). The corresponding cell is initially shared in common with the first group (cyan cell). This explains its marginal behavior that offers a compromise between the nonoscillatory state of the second group and the oscillatory state of the first.
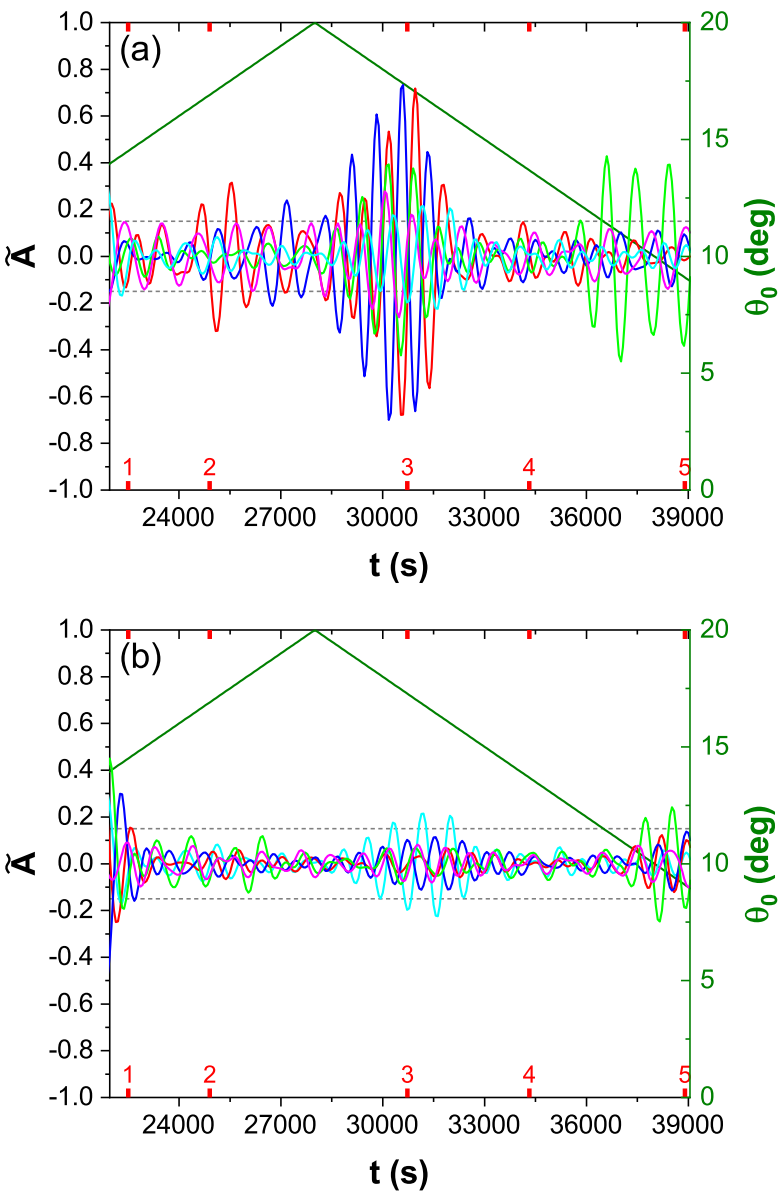

FIG. 14. Time evolution of the normalized cell surface areas $\tilde{A}(t)$ (curves) for (a) the unstable group of cells shown in Fig. 13(a), and (b) the stable group of cells shown in Fig. 13(b). The five times represented in the temporal sequences of Fig. 13 are marked by inner ticks labeled from 1 to 5 . Inclination angle $\theta_{0}$, straight line.

According to the previous remarks, one can infer that the local rearrangements that occur in the cellular pattern induce temporal variations of the cell-to-cell couplings in a given group. The cell oscillation periods are not much affected by these changes as already noticed but the oscillation amplitudes and phases are much more sensitive to the details of the couplings, thus to the local symmetries. This sensitivity is probably at the origin of the coexistence of the oscillatory and nonoscillatory dynamics of cell groups, and thus to the bursts observed here.

\section{SUMMARY AND DISCUSSION}

In the present paper, we have combined new experimental and numerical results to characterize more accurately the oscillatory-nonoscillatory transition in cellular patterns resulting from the solidification of a binary alloy under purely diffusive conditions.

The experimental study has been described in Sec. II. Directional solidification experiments using a succinonitrilecamphor alloy have been carried out in the DECLIC-DSI facility installed on board the International Space Station as part of a joint research program between CNES and NASA. 
The diffusive growth mode provided by the microgravity conditions allows to observe the dynamics of extended oscillating cellular patterns. Oscillations, observed in a narrow range of growth parameters, are manifested as a periodic variation of the apparent area of cells under top-view interface observation. We deepen here the analysis of two particular experiments $\left(V_{p}=0.75\right.$ and $\left.1.5 \mu \mathrm{m} / \mathrm{s}, G=19 \mathrm{~K} / \mathrm{cm}\right)$, characterized by the existence of two subgrains with different misorientations with respect to the thermal gradient, where the oscillation is only transient in one of the subgrains while it continues in the other. The experiments demonstrate a threshold for the drift velocity of the pattern beyond which oscillations are inhibited. This leads us to propose a criterion for the oscillation inhibition that is based on the ratio $\rho$ between the characteristic drift time and the oscillation period. We find that the threshold value that corresponds to the moment when the oscillations stop falls within the range $\rho_{\text {stop }}^{\exp } \simeq 2.3-2.8$.

The numerical study has been presented in Sec. III. It is focused on the effect of the misorientation angle $\theta_{0}$ on the cellular pattern dynamics (oscillatory vs nonoscillatory). In the simulations, $\theta_{0}$ is varied linearly in time, first increasing, then decreasing. We consider systems with different shapes and sizes to compare thin versus thick samples and narrow versus wide ones. In both cases, we find that increasing the system dimensions favors the onset of oscillations, especially in thin samples. Altogether, we find that the oscillation period is little affected by global and local geometrical constraints but that it is not the case for oscillation amplitudes, phase shifts, and velocity drifts that show significant sensitivity to theses constraints. As a result, bursts of oscillatory cell groups are observed to appear in bulk systems, even for the highest misorientation that definitely favors nonoscillatory behavior. Although a direct comparison between the experimental and numerical studies cannot be made because the corresponding control parameters differ, we obtain a reasonable agreement between the oscillatory-nonoscillatory thresholds $\rho_{\text {stop }}^{\exp } \simeq 2.3-2.8$ and $\rho_{\text {stop }}^{\text {num }} \simeq 1.4$ in bulk systems.

A few points have not been mentioned in the body of the paper that nevertheless warrant further study and/or discussion. First, the existence of a well defined threshold value for $\rho$ is legitimately questionable. The transition from a dynamical state to another one necessarily involves a characteristic time that is required by the concentration field to settle cooperative concentration fluxes between neighbor cells or to turn them uncooperative. If a threshold can still be defined, then it cannot be a static threshold as it is the case in most phase transitions. Also, a threshold must be defined statistically, which requires averaging it over many simulations/experiments. This preliminary study obviously does not fulfill the last requirement, so our results for $\rho_{\text {stop }}$ are rather rough estimates of the threshold. Second, there exists another threshold, $\rho_{\text {res }}$, that corresponds to oscillations resuming, after the nonoscillatory intermediate regime, when the misorientation angle is decreased. At first glance one can expect that $\rho_{\text {res }}=\rho_{\text {stop }}$. This would be true for a static threshold but, as just discussed, we rather have a dynamic threshold here. One additional dynamical effect is that the cell growth axes are progressively tilting towards the drift direction when $\theta_{0}$ is increased, while they are rotating opposite to the drift direction when $\theta_{0}$ is decreased. From our simulation results, we obtain $\rho_{\text {res }}^{\text {num }} \simeq 2.3$ that is close to $\rho_{\text {stop }}^{\exp } \simeq 2.3-2.8$.

In conclusion, our experimental and numerical results have combined to suggest the following picture of the physical mechanism for the oscillatory-nonoscillatory transition. Misorientation induces an overall drift of the cellular pattern, so the advective motion of the liquid phase toward the cells modifies the concentration fluxes between neighbor cells. At sufficiently high drift velocities, the flux balance that stabilizes the oscillatory state is so perturbed that the system stops oscillating (at least locally). The reverse is true when the drift velocity (misorientation) decreases.

\section{ACKNOWLEDGMENTS}

This research was achieved thanks to the support of CNES through the MISOL3D project (MIcrostructures de SOLidification 3D), NASA through Grants No. NNX12AK54G and No. 80NSSC19K0135 and Région PACA through the ENEMS project (Etude Numérique et Expérimentale des Microstructures de Solidification).
[1] P. E. Cladis, J. T. Gleeson, P. L. Finn, and H. R. Brand, Phys. Rev. Lett. 67, 3239 (1991).

[2] M. Ginibre, S. Akamatsu, and G. Faivre, Phys. Rev. E 56, 780 (1997).

[3] S. Akamatsu, S. Bottin-Rousseau, M. Perrut, G. Faivre, V. T. Witusiewicz, and L. Sturz, J. Cryst. Growth 299, 418 (2007).

[4] M. Georgelin and A. Pocheau, Phys. Rev. Lett. 79, 2698 (1997).

[5] J. Ghmadh, J. M. Debierre, M. Georgelin, R. Guérin, and A. Pocheau, Phys. Rev. E 98, 052802 (2018).

[6] N. Bergeon, D. Tourret, L. Chen, J.-M. Debierre, R. Guérin, A. Ramirez, B. Billia, A. Karma, and R. Trivedi, Phys. Rev. Lett. 110, 226102 (2013).

[7] D. Tourret, J. M. Debierre, Y. Song, F. L. Mota, N. Bergeon, R. Guérin, R. Trivedi, B. Billia, and A. Karma, Phys. Rev. E 92, 042401 (2015).
[8] J. Pereda, F. L. Mota, L. Chen, B. Billia, D. Tourret, Y. Song, J.-M. Debierre, R. Guérin, A. Karma, R. Trivedi, and N. Bergeon, Phys. Rev. E 95, 012803 (2017).

[9] C. Misbah, Dynamique complexe et morphogénèse (Springer, Paris, 2011).

[10] S. Gurevich, A. Karma, M. Plapp, and R. Trivedi, Phys. Rev. E 81, 011603 (2010).

[11] K. Kassner, C. Misbah, H. Müller-Krumbhaar, and A. Valance, Phys. Rev. E 49, 5477 (1994).

[12] C. Misbah and A. Valance, Phys. Rev. E 49, 166 (1994).

[13] K. Kassner, J.-M. Debierre, B. Billia, N. Noël, and H. Jamgotchian, Phys. Rev. E 57, 2849 (1998).

[14] M. Plapp and M. Dejmek, Europhys. Lett. 65, 276 (2004).

[15] J. Ghmadh, J.-M. Debierre, J. Deschamps, M. Georgelin, R. Guérin, and A. Pocheau, Acta Math. 74, 255 (2014). 
[16] J. Deschamps, M. Georgelin, and A. Pocheau, Phys. Rev. E 78, 011605 (2008)

[17] S. Akamatsu, G. Faivre, and T. Ihle, Phys. Rev. E 51, 4751 (1995).

[18] N. Bergeon, A. Ramirez, L. Chen, B. Billia, J. Gu, and R. Trivedi, J. Mater. Sci. 46, 6191 (2011).

[19] R. Marcout, G. Raymond, B. Martin, G. Cambon, B. Zappoli, F. Duclos, S. Barde, D. Beysens, Y. Garrabos, C. Lecoutre, B. Billia, N. Bergeon, and N. Mangelinck, in Proceedings of the 57th International Astronautical Congress (Valencia, Spain, 2006).

[20] F. L. Mota, N. Bergeon, D. Tourret, A. Karm, R. Trivedi, and B. Billia, Acta Mater. 85, 362 (2015).

[21] C. Weiss, N. Bergeon, N. Mangelinck-Noel, and B. Billia, Phys. Rev. E 79, 011605 (2009).

[22] J. Pereda, Y. Song, F. L. Mota, B. Billia, J.-M. Debierre, R. Guerin, A. Karma, R. Trivedi, and N. Bergeon, in Proceedings of the 67th International Astronautical Congress (Guadalajara, Mexico, 2016).

[23] Y. Song, S. Akamatsu, S. Bottin-Rousseau, and A. Karma, Phys. Rev. Materials 2, 053403 (2018).
[24] A. Karma and W. J. Rappel, Phys. Rev. E 53, 3017 (1996).

[25] A. Karma and W. J. Rappel, Phys. Rev. E 57, 4323 (1998).

[26] A. Karma, Phys. Rev. Lett. 87, 115701 (2001).

[27] B. Echebarria, R. Folch, A. Karma, and M. Plapp, Phys. Rev. E 70, 061604 (2004).

[28] A. K. Boukellal, J.-M. Debierre, G. Reinhart, and H. Nguyen Thi, Materialia 1, 62 (2018).

[29] A. K. Boukellal, A. K. Sidi Elvalli, and J.-M. Debierre, J. Cryst. Growth 522, 37 (2019).

[30] K. Glasner, J. Comput. Phys. 174, 695 (2001).

[31] A. Yamanaka, T. Aoki, S. Ogawa, and T. Takaki, J. Cryst. Growth 318, 40 (2011).

[32] J.-M. Debierre, R. Guérin, and K. Kassner, Phys. Rev. E 88, 042407 (2013).

[33] J. J. Hoyt, M. Asta, and A. Karma, Phys. Rev. Lett. 86, 5530 (2001).

[34] D. Tourret and A. Karma, Acta Math. 82, 64 (2015).

[35] J.-M. Debierre, R. Guérin, and K. Kassner, Phys. Rev. E 94, 013001 (2016). 